\title{
CANCER OF THE STOMACH.
}

\author{
By RONALD W. RAVEN, F.R.C.S.
}

(Surgeon to Out-Patients, French Hospital; Assistant Surgeon to Gordon Hospital for Rectal Diseases; Surgical Registrar, Royal Cancer Hospital (Free).)

The stomach is one of the organs in the body most frequently attacked by cancer. In the male it is the most frequent site of cancer and in the female it ranks third after the breast and organs of reproduction. Reference to the Registrar General's Statistical Review of England and Wales for the year I934 shows the following deaths from cancer of various sites.

Forms of Fatal Carcinoma of each Site-I934. SITE.

NUMBER OF DEATHS.

$\begin{array}{llccr}\text { All Sites } & \ldots & \ldots & 25,709 & 29,523 \\ \text { Stomach } & \ldots & \ldots & 6, \text { IOI } & 5,245 \\ \text { Uterus } & \ldots & \ldots & - & 4,033 \\ \text { Ovary } & \ldots & \ldots & - & \text { I,303 } \\ \text { Breast } & \ldots & \ldots & 50 & 6, \text { I99 } \\ \text { Rectum } & \ldots & \ldots & 2,98 \mathrm{I} & \mathrm{I}, 893 \\ \text { Colon } & \ldots & \ldots & 2,242 & 2,897\end{array}$

It is seen in the above table that carcinoma of the stomach accounted for II, 346 deaths out of a total of 55,232 deaths from carcinoma of all sites, that is 20 per cent. of the population died from carcinoma of the stomach in I934.

\section{THE EARLY LESION.}

An important contribution concerning the early pathology of cancer of the stomach was made by Versé in I908. He reported twelve early gastric carcinomata. All were adeno-carcinomata, 0.5 to $5 \mathrm{cms}$. diameter, slightly elevated, sometimes ulcerating, infected and infiltrated with leucocytes. In several cases there was a history of alcoholism and general atrophic or hyperplastic gastritis. Adenocarcinoma of the stomach may also develop from multiple foci separated by normal mucous membrane. Ewing calls attention to a group of cases in which the superficial epithelium and the epithelium of the ducts remain intact but the fundi of the tubular glands break up and these malignant epithelial cells infiltrate the mucosa over a wide area. This results in a superficial eroding carcinoma without tumour formation or excavation.

Carcinoma arises very infrequently from chronic hypertrophic gastritis. In the polypoid form of chronic gastritis however, the natural termination is the development of carcinoma. A similar series of changes may be found in the colon. Some authors consider that some proportion of cases of carcinoma of the stomach develop from heterotopic islands of intestinal mucosa which are frequently found in the pyloric region. Similarly heterotopic islands of pancreatic tissue may be the source of rare types of carcinoma of the stomach. 


\section{THE EARLY SYMPTOMS.}

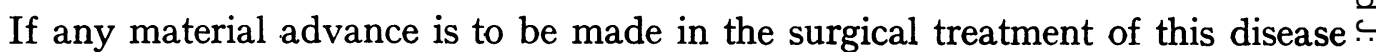
it is essential that the diagnosis should be reached in the early stages whilst the $\overrightarrow{\bar{\omega}}$ growth is still operable. In many of the text-books the symptoms detailed for 0 carcinoma of the stomach are those of the complications which have supervened $\frac{}{5}$ such as ulceration, pyloric obstruction or secondary injection and consequently $\frac{\vec{\Phi}}{\vec{\Phi}}$

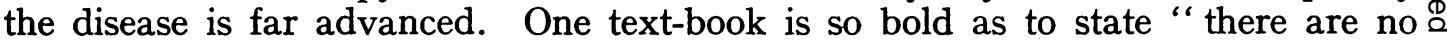
early symptoms." Late manifestations are nausea, vomiting, abdominal pain, is anæmia, marked loss of weight and a filling defect in the stomach on X-ray $\overrightarrow{0}$ examination. The following are important early symptoms and their manifesta- $\overrightarrow{\vec{\omega}}$ tion in a patient over 30 years of age should lead to a full investigation of the stomach.

Anorexia. In many patients, if a careful history is elicited, it is found that $\overrightarrow{\vec{\omega}}$ a gradual loss of appetite is an early symptom and its importance is increased if $\vec{A}$ other symptoms are associated with it. This loss of appetite is progressive until $\vec{i}$ there is repugnance of food.

Malaise. The patient with carcinoma of the stomach complains of becoming $\stackrel{\rho}{\rightarrow}$ easily fatigued and lack of a feeling of well-being. Often the symptoms in this $屯$ connection cannot be accurately described but he is conscious that something is wrong.

Abdominal discomfort. A frequent early symptom is vague abdominal dis comfort experienced in the upper half of the abdomen. This may be attributed by the patient to flatulent distension. There may be remissions for several months during which the patient is free from discomfort. As the disease progresses the discomfort becomes more marked until there is constant abdominal pain.

Nausea. This is frequently one of the first symptoms of carcinoma of the stomach. It is commonly experienced early in the morning before food is taken. Sometimes it may be noticed after meals.

Change in bowel habits. A change in bowel habits in a patient of cancer age should always lead to a thorough investigation of the gastro-intestinal tract. In carcinoma of the stomach constipation may be an early symptom. In the later stages of the disease when achlorhydria has developed there may be diarrhœea.

Pallor. A slight degree of pallor is frequently noticed during the early stages of carcinoma of the stomach. The pronounced pallor or lemon tint seen in these patients indicates the later stages when the disease is far advanced.

Loss of weight. In many cases there is a persistent and gradual loss of weight in the early stages and this is an important early. symptom.

\section{THE LATE SYMPTOMS.}

Abdominal pain. This symptom is frequently preceded by abdominal discomfort extending over a period of weeks or months. Again in certain cases there is no abdominal pain during the whole course of the disease. In the majority of $\frac{\mathbb{D}}{2}$ patients there is pain of varying severity. The character is dull aching and it $\stackrel{\mathbb{Q}}{2}$ 
is usually constant. It may be experienced during the night. It is frequently not related to food and situated in the epigastrium either to the right or left of the mid line according to the situation of the neoplasm. Sometimes the pain simulates that of peptic ulcer and sometimes it occurs in sharp paroxysms simulating biliary colic.

Vomiting. This is usually a late manifestation but in certain cases it may be an early symptom. It is generally the accompaniment of a neoplasm situated at the pylorus causing stenosis and obstruction. In these cases there may be varying degrees of dilatation of the stomach and the vomitus consists of large quantities of stagnating food which has accumulated over a day or more. The vomiting may occur at night in order to relieve the pain of gastric distension.

Hæmatemesis. This is seen in cases where the carcinoma has become ulcerated with erosion of the blood vessels of the stomach. The hæmorrhage varies from small quantities to large amounts causing death. The vomitus has the well-known " coffee ground" appearance.

Melæna. Tarry stools are sometimes seen in cases of carcinoma of the stomach and like hæmatemesis, this is generally a late symptom.

Cachexia. When the cachexia of malignant disease manifests itself it is safe to assume that the disease is very advanced.

Diarrhœa. This is sometimes a late symptom and usually accompanies achlorhydria.

Tumour. The presence of a tumour in the epigastrium in a patient with carcinoma of the stomach means that the disease is well established. It does not indicate however, that the disease is beyond eradication by surgery.

\section{THE DURATION OF SYMPTOMS.}

The duration of symptoms before patients with carcinoma of the stomach present themselves for treatment is short in the majority of cases. If the duration of symptoms is studied in a large series of cases it will be found that about onethird of the patients seek advice after symptoms have persisted for three months and under; about one-half after symptoms lasting six months and under.

In a small group of cases the disease runs a rapid course. In this fulminating variety the symptoms are present for only a few weeks and the disease is far advanced when the patient is first seen. If this small number of cases is excluded, it is found that the duration of symptoms has little influence on the operability of the carcinoma, thus, if the duration of symptoms is long this does not necessarily mean an inoperable carcinoma.

\section{LABORATORY AIDS TO DIAGNOSIS.}

Gastric analysis. Achlorhydria-It was formerly considered that achlorhydria was pathognomonic of carcinoma of the stomach. It is now known that achlorhydria occurs in other conditions such as pernicious anæmia and affections of the gall bladder. Not infrequently hyperchlorhydria is found in cases of carcinoma of the stomach. When achlorhydria accompanies carcinoma of the stomach it is a late manifestation of the disease. 
The presence of blood-The presence of occult blood in the resting contents of the stomach is of value in the early diagnosis of the disease. It must be remembered however, that blood may be.found in the contents of the stomach in cases of chronic peptic ulceration.

Lactic acid-In the late stages of carcinoma of the stomach the amount of lactic acid in the stomach contents is increased. This is due to a deficiency or absence of hydrochloric acid which usually inhibits the growth of lactic acid forming organisms. An increase in the lactic acid in the stomach is seen in cases of pyloric obstruction and dilatation of the stomach and is of no importance in the diagnosis of carcinoma of the stomach.

The Oppler-Boas Bacillus-This bacillus is associated with lactic acid and occurs in any condition in which there is achlorhydria. It is often found in the stomach contents of patients with gastric carcinoma but since it is also frequently encountered in non-malignant conditions it has no significance in the diagnosis of carcinoma of the stomach.

Examination of the stool. The presence of occult blood in the stool is an important aid in the diagnosis of the early case. In a series of 68 cases with carcinoma of the stomach I found that the reaction for occult blood in the stool was positive in 86 per cent. If the test is negative the diagnosis of carcinoma of the stomach is not excluded.

Examination of the blood. As the disease progresses a marked secondarye anæmia develops but the diagnosis must be made before these changes occur in the blood. In certain cases however, a profound anæmia is seen which resembles pernicious anæmia, before the definite diagnosis of carcinoma of the stomach is reached.

\section{TREATMENT.}

If a patient is believed to be suffering from carcinoma of the stomach after careful investigations have been carried out, an exploratory laparotomy should be performed without delay. In some of the early cases the diagnosis has to be made by this method.

The following points are noted at exploration in order to assess the operability of the neoplasm:-

(a) The site of the carcinoma-The most favourable site of carcinoma for $\frac{D}{3}$ excision is at the pylorus. The chances of cure diminish as the neoplasm N approaches the cardiac orifice.

(b) Fixity of the carcinoma-This may be due to carcinomatous infiltration or inflammatory adhesions between the stomach, and liver, pancreas or transverse colon. If many inflammatory adhesions need dividing the operative mortality increases.

(c) Involvement of Lymph Nodes-Moderate enlargement of the regional lymph nodes does not contra-indicate radical surgery as the enlargement may be inflammatory in nature. If the lymph nodes are of large size, hard in consistency, adherent and widespread it is generally unwise to attempt radical removal. 
(d) Nodules on the Peritoneum-The peritoneum must be inspected and the pelvis must be examined. If numerous small secondary nodules are found studded on the peritoneum the case is hopeless. If an isolated nodule is found in the pelvis this need not necessarily contra-indicate partial gastrectomy for a considerable period of relief may be experienced and the single nodule in the pelvis may be of slow growth.

(e) Other Viscera-The liver must be carefully examined for metastatic deposits. Both lobes should be palpated both superiorly and inferiorly. If the anterior portion of the liver is involved by direct extension of the growth this can be excised. An isolated metastasis in the liver need not necessarily preclude partial gastrectomy for there is evidence that life is prolonged and made much more bearable by palliative partial gastrectomy even with a single metastasis in the liver. The pancreas may be invaded by direct extension of the growth and the outlook in these cases is distinctly unfavourable. If the transverse colon is invaded by carcinoma of the stomach, resection of the involved segment of bowel may be performed - the mortality from operation is high in these cases.

Pre-operative preparation of the patient. It is essential to carry out certain preliminary preparations before operation for carcinoma of the stomach is performed. Anæmia, so often present in these çases, should be corrected by blood transfusions carried out over a period preceding operation. If the patient is dehydrated normal saline with 5 per cent. glucose should be administered by the rectal, subcutaneous or intravenous routes. If the carcinoma is ulcerating or fungoid giving rise to foul stomach contents gastric lavage may be carried out in an attempt to make the stomach cleaner. In these cases the administration of 0.5 per cent. solution of hydrochloric acid is of value.

The choice of operation. The choice of operation depends upon the extent of the disease and the site of the carcinoma. If the growth is operable it is necessary to perform an extensive resection of the stomach together with the regional lymphatics. As the great omentum is occasionally involved as well as the lesser omentum it is wise to remove both these structures. The greater portion of the lesser curvature must be excised as the disease tends to spread in the submucosa along the lesser curvature to the cardiac orifice. At least one inch of the first part of the duodenum should be removed owing to the possibility of permeation from the growth in the stomach. The operation which fulfils these requirements is partial gastrectomy after the method of Polya.

If the patient is emaciated and feeble owing to a carcinoma situated in the region of the pylorus causing pyloric obstruction it is wise to perform the operation in two stages. The first stage consists in gastro-jejunostomy on the proximal side of the growth. It is found that considerable improvement follows this procedure. At the second operation resection of the growth is carried out and the openings in the stomach and duodenum are closed.

If there is extensive infiltration of the stomach or if the carcinoma is situated at the cardia total gastrectomy should be performed provided there are no metastases in other viscera such as the liver. Careful pre-operative treatment should be instituted and proper preparations made in order to eliminate as much 
of the risk as possible. It is probable that this operation will be performed more frequently as technique advances. Palliative operations such as gastrojejunostomy, gastrostomy and jejunostomy are sometimes indicated in cases of carcinoma of the stomach. The usefulness of gastro-jejunostomy in carcinoma of the stomach is limited. The relief obtained is only partial as the infected, ulcerating growth is not removed. The average expectation of life after this operation is not more than about seven months. If, on the other hand, the growth is removed, even though there are metastases, the condition of the patient improves and life is more comfortable and may be prolonged for a year or more.

Irradiation in carcinoma of the stomach. The indication for this form of treatment is an inoperable growth, due to the extent of the disease, or the localization of the disease at the cardiac end of the stomach. It has been estimated that Io per cent. of carcinomata of the stomach are radio-sensitive and in practice it is found that a larger percentage will respond in varying degrees to irradiation. As a rule carcinoma of the cardiac and pyloric regions are more radio-sensitive than in other situations in the stomach.

Irradiation may be given from an external source - an X-ray tube or a radium applicator containing a large amount of radium. Interstitial irradiation has been employed in certain cases, especially if the carcinoma is situated at the cardiac end of the stomach: a greater dose can be given directly to the tumour and less $\bar{\omega}$ to neighbouring tissues than in external irradiation. For this purpose radon seed are implanted into the growth, the dosage depending on the size of the tumoure. The immediate results in certain cases of inoperable carcinoma of the cardiac en f $^{3}$ of the stomach treated with interstitial radon seeds are encouraging. Interstitial irradiation may be supplemented by external irradiation at a later date if necessary. 